Rudolf STRÄßER, JLU Gießen, ACU Brisbane

\title{
Educational Interfaces between Mathematics and Industry eine ICMI-Studie
}

\section{ICMI-Studien: Ablauf}

ICMI-Studien, also Studien, die von der Internationalen Mathematik Unterrichts Kommission IMUK (engl.: ICMI) angeregt werden, laufen nach einem vorgegebenen Muster ab: Nach Berufung eines Internationalen Programmkomitees („IPC“) erstellt dieses ein Diskussionsdokument (,DD“), welches dann möglichst breit publiziert wird, jedenfalls aber in der IMUKZeitschrift „Enseignement Mathématique“ und auf Anfrage im ZDM (für die EIMI-Studie vgl. Damlamian\&Sträßer 2009). Interessierte KollegInnen senden dann Beiträge an das IPC, das eine Auswahl von Beiträgen zum DD trifft und die AutorInnen zu einer Konferenz (,study conference") einlädt. Aufgrund der Konferenz und weiterer Beiträg wird dann ein Buch zur Studie (,study book") produziert, das in der Reihe „New ICMI Study Series (NISS)“ erscheint (für die EIMI-Studie vgl. Damlamian u.a. 2012).

\section{ICMI-ICIAM-Studie 20: Thematik}

Im Jahre 2009 starteten die ICMI und die International Conference for Industrial and Applied Mathematics (ICIAM) gemeinsam eine Studie mit dem Titel „Educational Interfaces between Mathematics and Industry (EIMI)“. Motiv für diese erste -Studie von ICMI in Kooperation mit anderen Institutionen war die Bedeutung der Nutzung von Mathematik in der Industrie, in den Unternehemen, im öfffentlichen Sektor und den alltäglichen Verrichtungen der BürgerInnen, um den Lebensunterhalt zu sichern. Dabei wird von einem breiten Verständnis von Industrie ausgegangen: “... as any activity of economic or social value, including the service industry, regardless of whether it is in the public or private sector" (vgl. Damlamian\&Sträßer 2009). Mathematik umfaßt für die Studie nicht nur das, was in der Öffentlichkeit unter Mathematik verstanden wird, sondern auch die verborgene, unsichtbare Mathematik (vgl. hierzu Abschnitt 5).

\section{Erziehung - Industrie: Kontraste}

Bei der Durchführung der Studie zeigten sich große Unterschiede zwischen den beteiligten Personenengruppen. Besonders auffällig war die Verschiedenheit der je verwendeten Sprechweisen. Selbst innerhalb einzelner Personengruppen zeigte sich ein so spezifischer Sprachgebrauch, dass man sinnvollerweise von einem besonderen „Jargon“ sprechen kann. Als Bei- 
spiel sei auf das Wort „Institutionalisierung“ verwiesen, das in der französisch-sprachigen Mathematikdidaktik eine von der in der Industrie verbreiteten Bedeutung deutlich verschieden ist.

Überdeutlich ist auch die Verschiedenheit der Zeit-Horizonte zwischen Industrie und Erziehung. In der industrie setzt man eher auf kurze Zyklen und Zeit-Horizonte, in denen ein Ergebnis erreicht werden soll, während im Erziehungswesen in der Regel auf eine längerfristige Entwicklung - zum Teil über mehrere Jahre - gesetzt wird.

Tatsächlich ist auch die Mathematik in der Industrie und im Erziehungswesen durchaus unterschiedlich. Handelt es sich um die sichtbare Mathematik (vgl. Abschnitt 5), so geht es in der Industrie oftmals um fortgeschrittene Teildisziplinen der Mathematik, wie etwa um partielle Differentialgleichungen oder komplexe lineare Algorithmen. Demgegenüber sollen im Erziehungswesen meist elementare Mathematik-Kenntnisse und Fähigkeiten vermittelt werden.

\section{Mathematik - Industrie: Modellbildung}

Es soll hier an das bekannte Kreislauf-Modell der Modellbildungnnert werden, das für MathematikdidaktikerInnen wie Personen aus der Industrie dazu diente, die Verwendung von Mathematik in industriellen Kontexten zu beschreiben und zu analysieren. Als Beleg aus der Industrie sei aus dem Hauptvortrag eines Mathematikers aus der Luftfahrt-Industrie zitiert: "The fundamental and familiar strategy is to use computer models to predict and optimize performance, validating these models with laboratory testing and analysis of existing products. In the aerospace industry the most promising approach is "multidisciplinary design optimization," or MDO. At one time our work in optimization dealt primarily with gradient methods for solving very large problems. An example is computing the minimum fuel trajectory to move a satellite from one stable orbit to another, a problem which might have 250,000 variables and 400,000 constraints" (Grandine at EIMI-studyconference).

In der Industrie ist die Modellbildung DER Weg in die Nutzung von Mathematik. Modellbildung wird mit einem strikt definierten Zweck außerhalb der Mathematik angewandt, um industrielle Probleme zu lösen. Mathematik ist Werkzeug. Demgegenüber ist die Modellbildung im Erziehungswesen ein wichtiges Ziel - man denke an die zentralen Kompetenzbereiche der "Standards". Tatsächlich hat sich die Modellbildung im Erziehungswesen manchmal bereits zu einem Unterrichtsgegenstand entwickelt, der um seiner selbst willen unterrichtet wird und die Tasache der „Verzweckung" der Mathematik eher verdeckt als öffentlich macht. Nach meiner 
Meinung muss in Mathematik-Unterricht in einer allgemeinbildenden Schule die Verzweckung der Mathematik bei der Modellbildung zeigen.

\section{Schwarze Kästen}

Als Illustration zu diesem Abschnitt sei an die Entwicklung des Wiegens und der Preisberechnung erinnert, wie sie sich in den nachstehenden drei Bildern ausdrückt (vgl. Straesser 2002; die Bilder wurden von der Firma BIZERBA zur Verfügung gestellt).
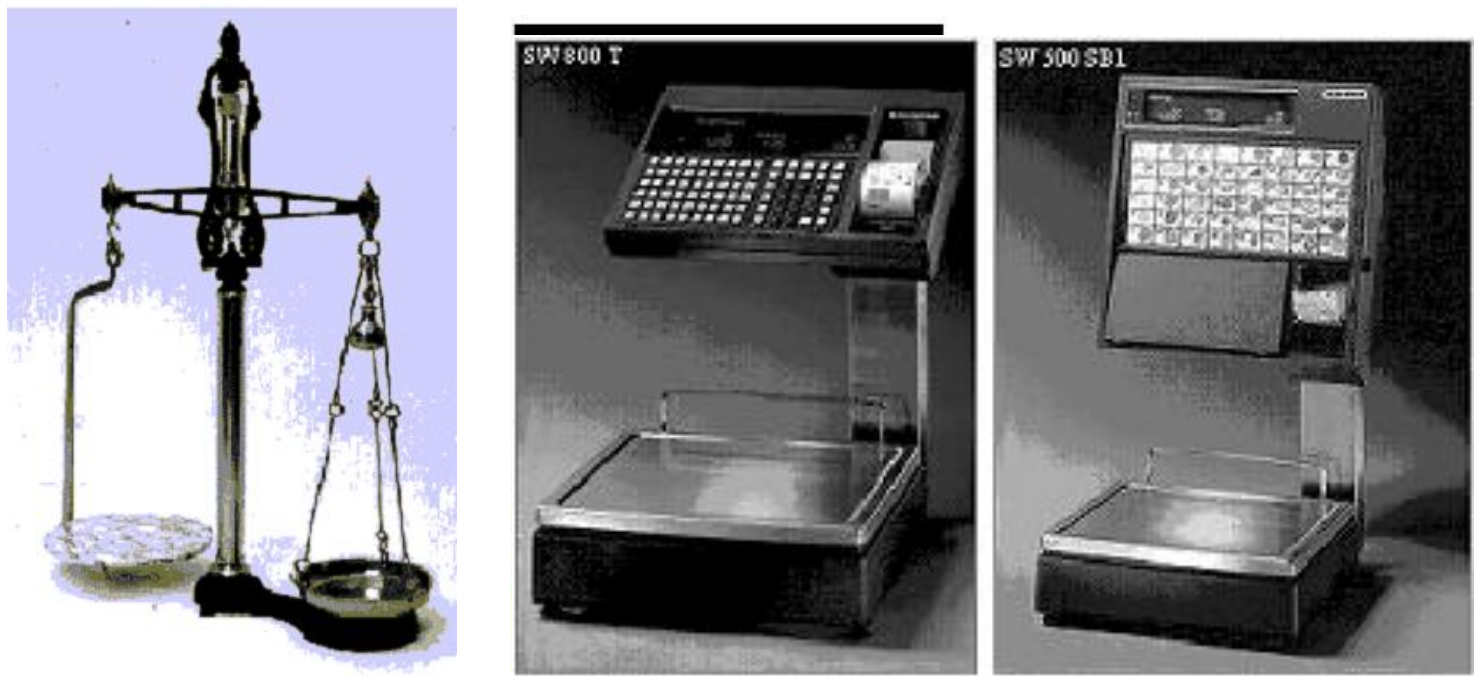

Eine Erklärung für das Verschwinden der Mathematik aus der öffentlichen Wahrnehmung ist die zunehmende Nutzung "Schwarzer Kästen“ (von „black boxes“) als Verpackung und Versteck der Mathematik. In der EIMIStudie wurden als Konsequenzen der zunehmenden Nutzung von Schwarzen Kästen benannt: Die Nutzung Schwarzer Kästen können industriellen Fortschritt behindern, weil die in ihm realisierte Problemlösung nicht mehr sichtbar und unzugänglich ist. Dies erschwert auch die Fehlersuche und die Fehlerbehebung. Wenn eine Problemlösung in einem Schwarzen Kasten versteckt ist, läßt sich diese Lösung in der Regel auch schwerer bewerten und kritisieren.

Für einen Lernprozess läßt sich eine ambivalente Wirkung der Nutzung von Schwarzen Kästen festhalten. Ein Schwarzer Kasten kann einerseits das Verständnis einer Problemlösung behindern, weil er die zugrundeliegenden Algorithmen verdeckt. Andererseits kann die Zusammenfassung einer Problemlösesequenz zu einer Einheit das Lernen dieser Problemlösung erleichtern (durch "encapsulation"). Zusätzlich fördert der Einsatz von Informationstechnologie den Einsatz Schwarzer Kästen und ermöglicht für Lehr/Lernprozesse die Simulation von Problemlösungen, die so ohne Gefahr für Material und Mensch eingeübt werden können. Man denke nur an die 
Simulation von Herstellungsprozessen unter Einsatz von CNCWerkzeugen.

\section{Persönlicher Schluß}

Die EIMI-Studie hat einige allgemeinere Tatsachen ans Licht gebracht, auf die ich abschließend hinweisen möchte. So wurde überdeutlich, dass es keine institutionalisierte Forschung zur Nutzung der Mathematik in der Industrie gibt. Forschung zu diesem Thema ist individuelles Hobby von Einzelpersonen, die oft diskontinuierlich nach den persönlichen und beruflichen Möglichkeiten betrieben wird. Eine bedauerliche Konsequenz dieser Tatsache ist, dass Personen, die in diesem Feld arbeiten, immer wieder das „Rad neu erfinden“. Es fehlt ein auch nur einigermaßen durch Praxis gesichertes Forschungsparadigma. Forschungsmethoden werden immer wieder den Nachbar-Disziplinen entliehen. Gegenstands-adäquate Forschungsmethoden sollten stattdessen langfristig entwickelt werden. Man kann nur hoffen, dass das 'Study Book' hier Fortschritte bringt

\section{Literatur}

Araújo, A., Fernandes, A., Azevedo, A., \& Rodrigues, J. F. (Hrsg.). (2010). Proceedings EIMI 2010 Conference. Educational Interfaces between Mathematics and Industry. Lisbon / Bedford, MA: CIM / Comap.

Damlamian, A., Rodrigues, J.-F. \& R. Sträßer (Hrsg.) (erscheint 2012). Educational interfaces between mathematics and industry. ICMI Study 20. Berlin Heidelberg: Springer.

Damlamian, A. - Straesser, R. (On behalf of the International Program Committee, 2009). ICMI-study 20: educational interfaces between mathematics and industry. ZDM Mathematics Education 41 (4), 525-533.

Organisation for Economic Co-operation and Development, Global Science Forum (2008) Report on Mathematics in Industry. http://www.oecd.org/dataoecd/47/1/41019441.pdf

Straesser, R. (2002). On the disappearance of Mathematics from society's perception. Developments in Mathematics Education in German-speaking Countries. Selected Papers from the Annual Conference on Didactics of Mathematics, Bern, 1999. H.G. Weigand u.a. (Hrsg.). Hildesheim - Berlin, Franzbecker: 124-133. 